pointe (paroxysmal ventricular tachycardia), and ictal asystole. Asystole has a role in sudden unexplained death in epilepsy.

Ictal asystole and anti-NMDAR antibody encephalitis. Ictal asystole is recently reported as a complication of anti-NMDAR encephalitis. In this 15-year-old girl, seizures with asystole developed 26 days after initial presentation of symptoms and temporal lobe seizures that were associated with bradycardia. After insertion of a demand pacemaker on day 46, there were no further cardiac events. (Millichap JJ, Goldstein JL, Laux LC, Nordli DR, Stack CV, Wainwright MS. Pediatrics 2011;127(3):e781-6). In this case, asystole occurred at the onset of the illness and was not explained by a prolonged recurrence of seizures.

\title{
FUNCTIONAL NEUROIMAGING IN STARTLE EPILEPSY
}

Researchers at the Epilepsy Unit, Hospital Clinic de Barcelona, Spain investigated brain areas involved in startle-induced seizures, using a functional neuroimaging approach in 4 adult patients whose seizures began at age 4 months to 10 years. Presurgical evaluation included ictal SPECT coregistered to MRI. Startle-induced seizures were bilateral asymmetric tonic with ictal-EEG pattern located over the mesial centroparietal region. Three patients had a significant hyperperfusion ( $>2 \mathrm{SD}$ above the reference) involving the supplementary motor area, the perirolandic area, and precuneus. Ictal EEG-fMRI showed an initial activation located over the precuneus, supplementary motor area, cingulate gyrus, and the precentral/perirolandic area. Startle-induced seizures triggered by unexpected stimuli are generated by the interaction of a frontoparietal network located over the mesial brain surface. (Fernandez S, Donaire A, Maestro I, et al. Functional neuroimaging in startle epilepsy: Involvement of a mesial frontoparietal network. Epilepsia Sept 2011;52(9):1725-1732). (Respond: Santiago Fernandez MD, Email: santiago.fernandez@hospitalplato.com).

COMMENT. Startle epilepsy is triggered by unexpected stimuli, generally a sudden noise, somatosensory, or visual stimuli. First described by Alajouanine and Gastaut (1955), and included in the ILAE classification 2001. A frontoparietal epileptic network is involved, and not a discrete focus.

\section{ATTENTION DEFICIT COMORBID DISORDERS}

\section{WRITTEN-LANGUAGE DISORDER AND ADHD}

Researchers at Mayo Clinic, Rochester, MN, and Children's Hospital Boston, MA evaluated the incidence rates of written-language disorder (WLD), with and without reading disability (RD), according to gender, among children with and without attention deficit hyperactivity disorder (ADHD) in a population-based birth cohort. The cumulative incidence of 'WLD by 19 years of age was significantly higher for children with ADHD than for children without ADHD, for both boys and girls (boys: $64.5 \%$ vs $16.5 \%$; girls: $57.0 \%$ vs $9.4 \%$ ). In contrast, among children without ADHD, the 